\title{
Observation of non-Gaussianity and phase synchronization in intermittent magnetic field turbulence in the solar-terrestrial environment
}

\author{
Rodrigo A. Miranda ${ }^{1,2}$, Abraham C.-L. Chian ${ }^{1,2}$, Sergio Dasso ${ }^{3}$, \\ Ezequiel Echer ${ }^{1}$, Pablo R. Muñoz ${ }^{1}$, Nalin B. Trivedi ${ }^{1}$, \\ Bruce T. Tsurutani ${ }^{4}$, and Michio Yamada ${ }^{2}$ \\ ${ }^{1}$ National Institute for Space Research (INPE), \\ São José dos Campos-SP 12227-010, Brazil \\ email: rmiranda@dge.inpe.br \\ ${ }^{2}$ Research Institute for Mathematical Sciences (RIMS), Kyoto University, \\ Kyoto 606-8502, Japan \\ ${ }^{3}$ Institute of Astronomy and Space Sciences, CONICET-University of Buenos Aires, \\ Buenos Aires, Argentina \\ ${ }^{4}$ Jet Propulsion Laboratory (JPL/NASA), California Institute of Technology, \\ Pasadena, CA, USA
}

\begin{abstract}
We apply two nonlinear techniques, kurtosis and phase coherence index, to analyze magnetic field measurements from SOHO MDI solar images, ACE and Cluster data in the solar wind, and ground magnetometers in Brazil. We focus on two events: a non-ICME event in February 2002 and an ICME event in January 2005. Finite degree of non-Gaussianity and phase synchronization are observed in all datasets. The nonlinear response of the Earth's geomagnetic field to an ICME event in the solar wind is discussed.
\end{abstract}

Keywords. turbulence, Sun: photosphere, solar wind

\section{Introduction}

The solar-terrestrial environment provides a natural laboratory for observing intermittent turbulence (Kamide \& Chian, 2007). The degree of non-Gaussianity (intermittency) in a turbulence can be quantified by calculating kurtosis (i.e., flatness minus 3), which is defined as the normalized fourth-order structure function based on two-point differences in space $(r)$ or time $(\tau)$ assuming Taylor hypothesis. Recently, a phase coherence surrogate technique for characterizing phase synchronization in turbulence based on the null hypothesis was developed for space plasmas (Hada et al., 2003). The link between non-Gaussianity and phase synchronization in intermittent turbulence was established by Koga et al. (2007) using the Geotail magnetic field data upstream and downstream of the Earth's bow shock. The phase coherence surrogate technique was used to study phase synchronization in magnetic field data from Cluster in the magnetosheath close to the Earth's magnetopause (Sahraoui et al., 2008), in plasma density fluctuations observed by SOHO image in the solar corona (Telloni et al., 2009) and in magnetic field data both in the shocked solar wind measured by Cluster upstream of the Earth's bow shock and in the unshocked ambient solar wind measured by ACE at the L1 Lagrangian point (Chian \& Miranda, 2009). 
In this paper, we study the scale dependence of kurtosis and phase coherence in intermittent magnetic field turbulence measured at three different locations of the solarterrestrial environment: (1) in the solar photosphere, (2) in the solar wind, and (3) on the ground. We investigate two scenarios: a non-ICME event in February 2002 and an ICME event in January 2005.

\section{Non-Gaussianity and phase synchronization in the solar-terrestrial environment}

Solar images obtained by the SOHO MDI instrument provide the measurement of the magnetic field in the solar photosphere. Near the centre of the solar image the projection effects are negligible, hence the MDI solar image represents the vertical or line-of-sight component of the photospheric magnetic field $B_{\|}$(Abramenko et al., 2002). Figure 1(a) shows a solar magnetogram taken by SOHO MDI on 1 February 2002. The white color corresponds to positive magnetic polarity, and the black color corresponds to negative magnetic polarity. The two white squares mark two selected areas, one containing the active region AR 09802 (upper) and the other containing a quiet region (lower). Figure 1(b)

SOHO MDI Solar image at 22:24 UT on 1 February 2002

(a)

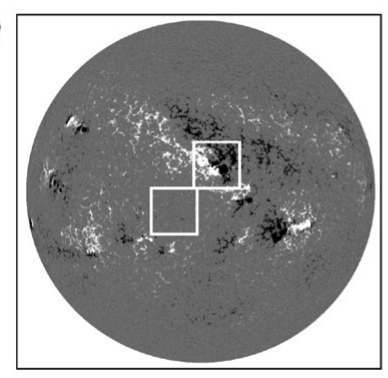

(b)

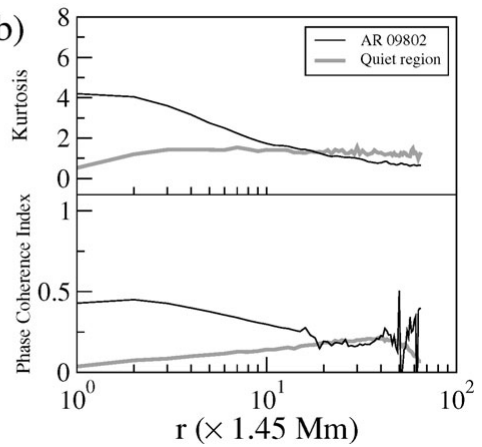

Figure 1. (a) SOHO MDI solar image taken at 22:24 UT on 1 February 2002. The two white squares enclose two areas containing the active region AR 09802 (upper), and a quiet region (lower). (b) Kurtosis (upper panel) and the phase coherence index (lower panel) as a function of spatial scale r computed from AR 09802 and the quiet region.

SOHO MDI Solar image at 04:47 UT on 16 January 2005

(a)

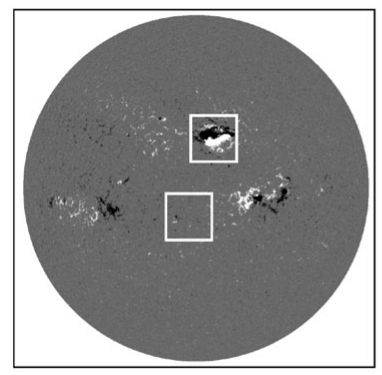

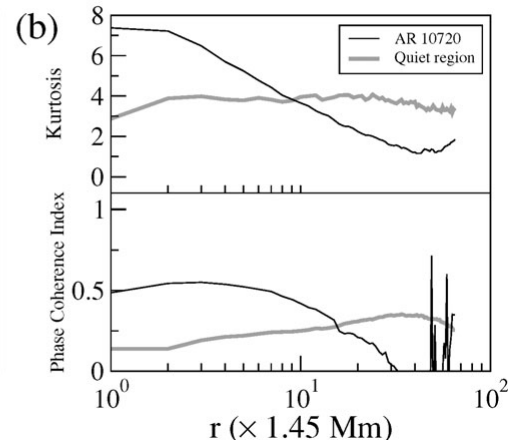

Figure 2. (a) SOHO MDI solar image taken at 22:24 UT on 16 January 2005. The two white squares enclose two areas containing the active region AR 10720 (upper), and a quiet region (lower). (b) Kurtosis (upper panel) and the phase coherence index (lower panel) as a function of spatial scale r computed from AR 10720 and the quiet region. 
shows kurtosis and the phase coherence index as a function of spatial scale $r$ computed from the two selected regions. From Figure 1(b) we observe that the variation of kurtosis with $r$ in the quiet region is close to a Gaussian process $(K=0)$, being scale-invariant for all scales $r \gtrsim 2$ pixels $\sim 2.9 \mathrm{Mm}$, consistent with the features of a monofractal process (Abramenko et al., 2002). The active region, on the other hand, displays an increase of kurtosis as the spatial scale $r$ decreases, which is a characteristic of a non-Gaussian process related to nonlinear energy cascade within the inertial subrange, and these values are higher than those obtained from the quiet region for scales $\lesssim 20$ pixels $\sim 29 \mathrm{Mm}$. The degree of phase synchronization measured by the phase coherence index (Hada et al., 2003; Koga et al., 2007; Chian \& Miranda, 2009) in the active region increases with decreasing spatial scale $r$, while the quiet region presents low-degree of synchronization at all scales. Note that at large scales the lack of datapoints introduces big errors in the computation of the phase coherence index.

Figure 2(a) shows a SOHO MDI solar magnetogram obtained on 16 January 2005. The two white squares enclose two selected areas containing the active region AR 10720 (upper) and a quiet region (lower), respectively. On 19-20 January 2005 several flares associated with CMEs were observed in AR 10720, however in this period the active region is too close to the solar limb, and the projection effects cannot be neglected. Hence, we restrict our analysis to this earlier solar image when AR 10720 is near the disk centre. Figure 2(b) shows kurtosis and the phase coherence index as a function of spatial scale $r$. It shows that the kurtosis of AR 10720 increases as the scale $r$ decreases, while kurtosis of the quiet region displays scale-invariance, similar to Fig. 1(b). Likewise, the phase coherence index in Fig. 2(b) presents similar behavior as Fig. 1(b).

Now we direct our attention to the interplanetary magnetic field data collected in situ in the solar wind. Figure 3(a) shows the time series of the modulus of magnetic field $|B|$ obtained by ACE and Cluster from 19:40:40 UT on 1 February 2002 to 03:56:38 UT on 3 February 2002. During this interval Cluster is in the solar wind upstream of the Earth's bow shock (Chian \& Miranda, 2009). Although Fig. 1(a) indicates the presence of several solar active regions on 1 February 2002, no M- or X-class solar flares occurred during the selected interval, and strong interplanetary disturbances such as ICMEs were not seen. The upper panel of Figure 3(b) shows the variation of kurtosis as a function of time scale $\tau$ for magnetic field fluctuations of ACE and Cluster. For $10 \mathrm{~s} \lesssim \tau \lesssim 10^{3} \mathrm{~s}$, kurtosis increases as the time scale decreases which characterizes non-Gaussianity. The lower panel of Fig. 3(b) shows the variation of the phase coherence index with $\tau$ which presents similar behavior as kurtosis. From Fig. 3(b) we observe that the behavior of kurtosis and the phase coherence index detected by ACE and Cluster are very similar except for scales around $10 \mathrm{~s}$, where ACE observes a higher level of intermittency and phase synchronization than Cluster.

Figure 4(a) shows the time series of the modulus of magnetic field $|B|$ measured by ACE for the ICME event of 21-22 January 2005 (Foullon et al., 2007). We selected two intervals from this event. The first interval is located upstream of the ICME shock which begins at 06:00:00 UT on 21 January and ends at 16:00:00 UT on 21 January. The second interval is located downstream of the ICME shock which begins at 16:47:19 UT on 21 January, and ends at 21:20:00 UT on 22 January. In order to ensure the stationarity of data we avoid the "foot" transition region associated with the ICME shock front. Figure 4(b) shows kurtosis and the phase coherence index as a function of time scale $\tau$ computed from the modulus of magnetic field $|B|$ in upstream and downstream regions of the ICME shock. It is evident, from Figure 4(b), that both upstream and downstream regions indicate the features of intermittency and phase synchronization across scales; moreover, the level of 
intermittency and phase synchronization are higher in the downstream region than the upstream region for all scales $\tau$.

We analyze next the Earth's geomagnetic field data obtained from two ground magnetometers. The top panel of Fig. 5(a) shows the time series of the modulus of magnetic field $|B|$ observed by ACE at L1 during the non-ICME solar wind event of 1-3 February 2002. The second panel shows the modulus of the Earth's geomagnetic field measured by a ground magnetometer at Ji-Paraná (JPA), Brazil (geomagnetic latitude $\sim 0^{\mathrm{O}}$ ). The three bottom panels show the time series of Pc3, Pc4 and Pc5 geomagnetic micropulsations, respectively. Each time series of geomagnetic micropulsations is obtained by applying a Fourier band-pass filter. Fig. 5(b) shows kurtosis and the phase coherence index of ACE magnetic field data and JPA geomagnetic field data as a function of time scale $\tau$. Fig. 5(b) indicates that the geomagnetic field fluctuations measured on the ground are intermittent. For almost all scales the level of intermittency and phase coherence of ACE are higher than the JPA.

Solar wind event of 1-3 February 2002

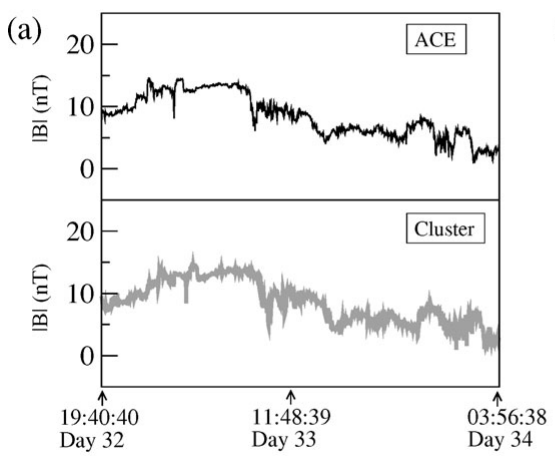

Time (UT)

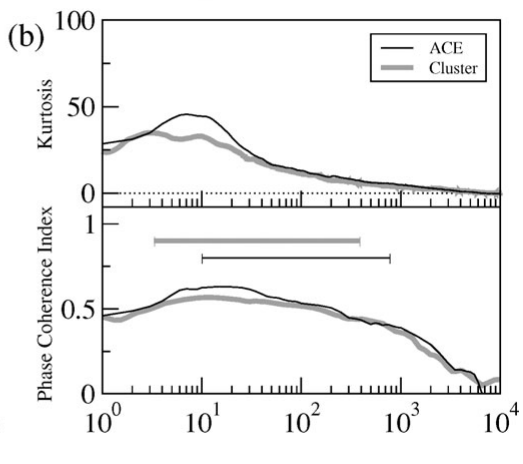

$\tau(\sec )$

Figure 3. (a) Time series of the modulus of magnetic field $|B|$ measured by ACE (upper panel) and Cluster (lower panel) for the solar wind event of 1-3 February 2002. (b) Kurtosis (upper panel) and the phase coherence index (lower panel) of $|B|$ as a function of time scale $\tau$. Horizontal bars indicate the inertial subranges of $\mathrm{ACE}$ and Cluster, respectively.

ICME event of 21-22 January 2005

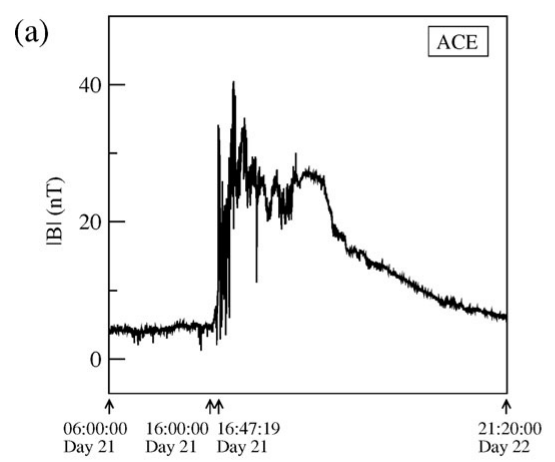

Time (UT)

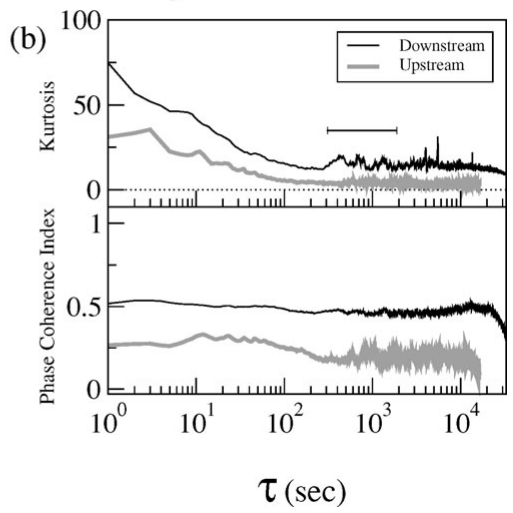

Figure 4. (a) Time series of the modulus of magnetic field $|B|$ measured by ACE for the ICME event of 21-22 January 2005. (b) Kurtosis (upper panel) and the phase coherence index (lower panel) of $|B|$ as a function of time scale $\tau$ for the upstream and downstream regions of the ICME shock. 
Ground magnetometer data at Ji-Paraná(JPA), Brazil

(Geomagnetic latitude: $\sim 0^{\circ}$ )
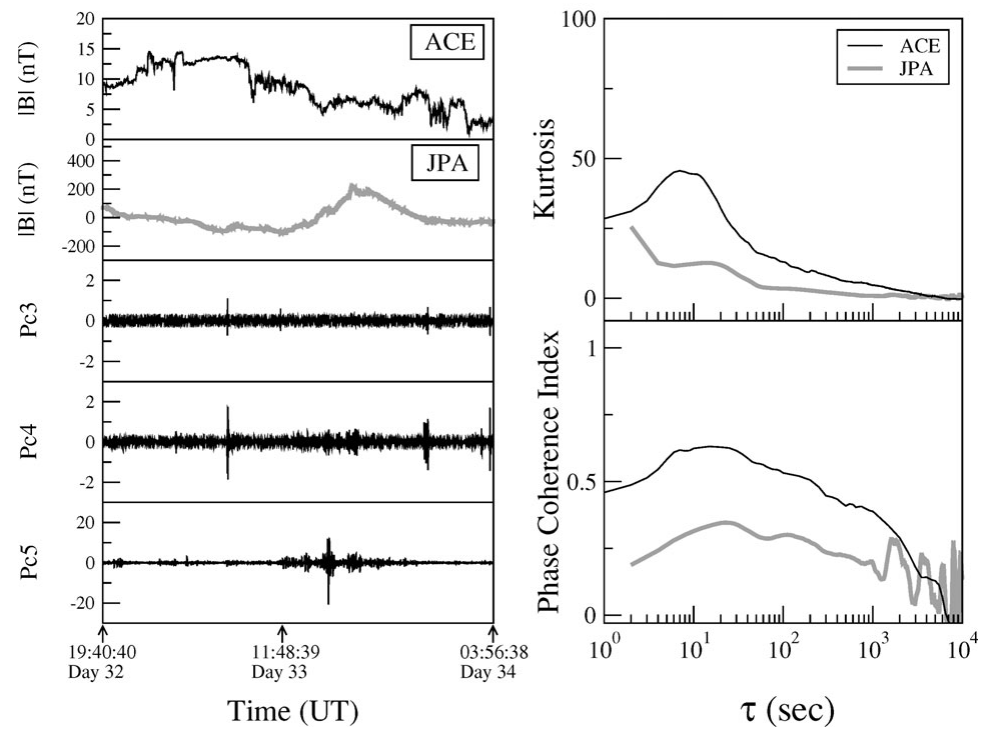

Figure 5. (a) From top to bottom: time series of $|B|$ measured by ACE for the solar wind event of 1-3 February 2002; modulus of the Earth's geomagnetic field $|B|$ measured by a ground magnetometer at Ji-Paraná, Brazil, during the same time interval; time series of Pc3 (10-45 s), Pc4 (45-150 s) and Pc5 (150-1000 s) micropulsations. (b) Kurtosis (upper panel) and the phase coherence index (lower panel) of $|B|$ measured by ACE and the ground magnetometer at JPA as a function of time scale $\tau$.

Ground magnetometer data at Vassouras (VSS), Brazil

(Geomagnetic latitude: 19 ${ }^{\circ}$ )

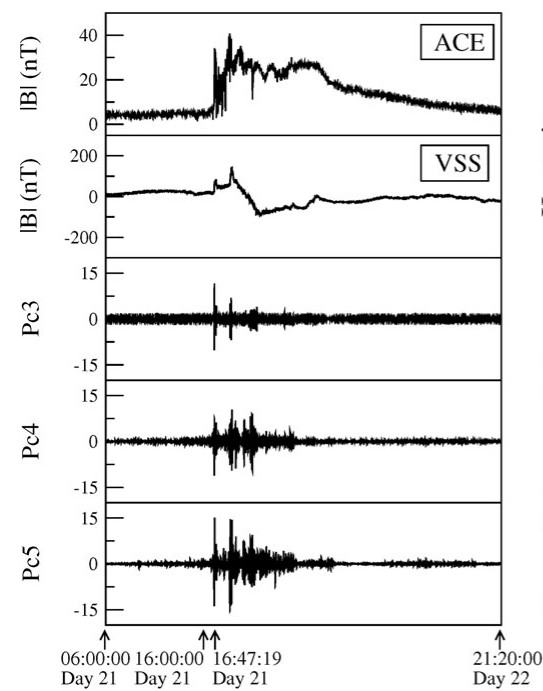

Time (UT)

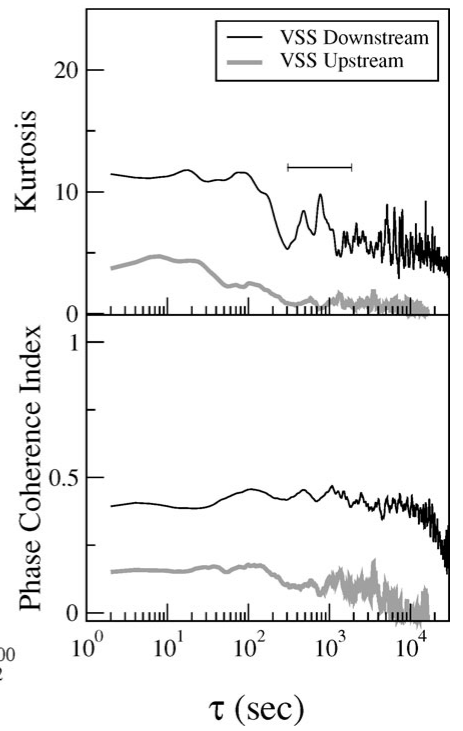

Figure 6. (a) From top to bottom: time series of $|B|$ (nT) measured by ACE for the ICME event of 21-22 January 2005; modulus of the Earth's geomagnetic field $|B|(\mathrm{nT})$ measured by a ground magnetometer at Vassouras, Brazil; time series of Pc3 (10-45 s), Pc4 (45-150 s) and Pc5 (150-1000 s) micropulsations. (b) Kurtosis (upper panel) and the phase coherence index (lower panel) of $|B|$ measured by ACE and the ground magnetometer at VSS as a function of time scale $\tau$. 
Finally, for the ICME event of 21-22 January 2005, we plot in the top panel of Figure $6(\mathrm{a})$ the time series of the modulus of magnetic field $|B|$ observed by ACE and in the second panel the modulus of the Earth's geomagnetic field measured by a ground magnetometer at Vassouras (VSS), Brazil (geomagnetic latitude $\sim 19^{\mathrm{O}}$ ). The three bottom panels show the time series of Pc3, Pc4 and Pc5 geomagnetic micropulsations. All the time series of VSS were shifted by $-1462 \mathrm{~s}$ to synchronize with the ICME shock arrival at ACE. After shifting, we divide the VSS geomagnetic field time series into "upstream" and "downstream" intervals in analogy with the ACE magnetic field data of the ICME shock. Figure 6(b) shows kurtosis and the phase coherence index of the VSS upstream and downstream intervals as a function of time scale $\tau$. It shows that for all scales the level of intermittency and phase coherence are higher in the "downstream" geomagnetic field-fluctuations after the arrival of ICME than the "upstream" geomagnetic field fluctuations before the arrival of ICME.

A comparison of the variation of kurtosis and the phase coherence index as a function of $\tau$ for the downstream intervals observed by ACE in the solar wind (Fig. 4(b)) and the VSS ground magnetometer (Fig. 6(b)) reveals a common feature consisting of three peaks between scales $\tau \sim 304 \mathrm{~s}$ and $\tau \sim 1860 \mathrm{~s}$, marked with a bar in both figures. This can be interpreted as evidence of a close correlation of the Earth's geomagnetic field with the ICME driver at these scales.

\section{Conclusions}

In this paper, we studied non-Gaussianity and phase synchronization in solar-terrestrial magnetic field turbulence in two scenarios: a non-ICME event and an ICME event. For each scenario, we calculated first kurtosis and the phase coherence index for an active region and a quiet region, respectively, in the solar photosphere. We then applied the same techniques to solar wind turbulence using in situ data. The response of the Earth's geomagnetic field using data from ground magnetometers in Brazil was presented. In particular, we showed that the interplanetary magnetic field turbulence downstream of the ICME shock is closely correlated with the geomagnetic turbulence detected on the ground.

\section{Acknowledgments}

R. A. Miranda and A. C.-L. Chian wish to thank Professor Michio Yamada, Dr. Yoshitaka Saiki and RIMS at Kyoto University for their kind hospitality. This work is supported by CAPES and CNPq, Brazil.

\section{References}

Abramenko, V. I., Yurchiyshiyn, V. B., Wang, H., Spirock, T. J., \& Goode, P. R 2002, ApJ 577, 487

Chian, A. C.-L. \& Miranda, R. A. 2009, Ann. Geophys. 27, 1789

Foullon, C., Owen, C. J., Dasso, S., Green, L. M., Dandouras, I., Elliott, H. A., Fazakerley, A. N., Bogdanova, Y. V., \& Crooker, N. U. 2007, Solar Phys. 244, 139

Hada, T., Koga, D., \& Yamamoto, E. 2003, Space Sci. Revs 107, 463

Kamide, Y. \& Chian, A. C.-L. (Eds.) 2007, Handbook of the Solar-Terrestrial Environment (Berlin, Springer)

Koga, D., Chian, A. C.-L., Miranda, R. A., \& Rempel, E. L. 2007, Phys. Rev. E 75, 046401

Sahraoui, F. 2008, Phys. Rev. E 78, 026402

Telloni, D., Antonucci, E., Bruno, R., \& D'Amicis, R 2009, ApJ 693, 1022 